\title{
Expression and significance of EBV, ARID1A and PIK3CA in gastric carcinoma
}

\author{
HUAN ZHOU, SHUN TAN, HONG LI and XIANGTAO LIN \\ Department of Pathology, Central South University, Xiangya School of Medicine, \\ Affiliated Haikou Hospital, Haikou, Hainan 570208, P.R. China
}

Received May 7,2018; Accepted December 14, 2018

DOI: $10.3892 / \mathrm{mmr} .2019 .9886$

\begin{abstract}
AT-rich interaction domain 1A (ARID1A) and phosphatidylinositol-4,5-bisphosphate 3-kinase catalytic subunit $\alpha$ (PIK3CA) serve important roles in the formation and development of numerous malignancies including gastric cancer. Accumulating evidence has demonstrated that Epstein-Barr virus (EBV) is a pathogenic virus associated with gastric cancer. The present study aimed to investigate the association between EBV infection, and the expression levels of ARID1A and PIK3CA in gastric cancer. EBER in situ hybridization was performed to detect EBV infection. Immunohistochemistry was used to assess the expression levels of ARID1A and PIK3CA in gastric cancer and adjacent normal tissues. A total of 58 gastric cancer and 10 adjacent normal tissues were tested for genetic mutations via single nucleotide polymorphism genotyping assays. Fluorescent polymerase chain reaction was used to detect EBV infection; 9.3\% (28/300) of gastric cancer samples were positive for EBV, whereas, all adjacent normal tissues were negative. ARID1A and PIK3CA were negatively correlated in gastric cancer $(r=-0.167)$. The expression levels of ARID1A and PIK3CA in gastric cancer were significantly associated with the depth of invasion of gastric cancer. A total of $62.1 \%$ (36/58) of tumor samples exhibited mutations in ARID1A, whereas, $13.8 \%(8 / 58)$ presented mutations in PIK3CA. Notably, EBV-associated gastric cancer (EBVaGC) samples with PIK3CA mutations additionally exhibited ARID1A mutations. Although in the present study it was identified that ARID1A and PIK3CA were negatively correlated in $\mathrm{EBVaGC}$, further studies are required to investigate the association among ARID1A, PIK3CA and $\mathrm{EBV}$ in gastric cancer.
\end{abstract}

Correspondence to: Professor Shun Tan, Department of Pathology, Central South University, Xiangya School of Medicine, Affiliated Haikou Hospital, 43 People's Avenue, Haikou, Hainan 570208, P.R. China

E-mail: 18774947230@163.com

Key words: gastric cancer, Epstein-Barr virus, AT-rich interaction domain 1A, phosphatidylinositol-4,5-bisphosphate 3-kinase catalytic subunit $\alpha$

\section{Introduction}

Gastric cancer is one of the most common types of cancer in the world, particularly in eastern countries such as China, Japan and Korea (1). Although the incidence of gastric cancer has decreased over the past years, gastric cancer remains the fourth most common type of cancer in the world with the second highest lethality rate. In total, $\sim 90 \%$ of patients with gastric cancer relapse within 3 years following surgery, and the prognosis remains poor (2). The pathologic mechanisms underlying gastric cancer are heterogeneous and include various environmental and genetic carcinogenic factors. The effect of Helicobacter pylori infections on gastric cancer had been an extensively discussed topic. In recent years, the focus has shifted to the role of Epstein-Barr virus (EBV) on gastric cancer. A recent study identified that $\sim 10 \%$ of patients with gastric cancer exhibited EBV infection (3). The Cancer Genome Atlas categorizes EBV-associated gastric cancer (EBVaGC) as a distinct type of gastric cancer, according to a novel molecular pathological classification (4). EBVaGC is a particular subtype of gastric cancer with distinct clinicopathological features; EBV antigenic epitopes have been detected specifically in gastric cancer cells, demonstrating that EBV may be a virulence factor in gastric cancer (5).

AT-rich interaction domain 1A (ARID1A) is located on chromosome 1p36.11 (6), and acts as a key component of the yeast SWItch/Sucrose Non-Fermenting (SWI/SNF) complex. Accumulating evidence has demonstrated that ARID1A is a tumor suppressor gene that was identified to be mutated in a variety of human tumors (7). ARID1A is able to interact with DNA and transcription factors, serving a role in chromatin remodeling (8). According to statistics, $\sim 20 \%$ of human cancers exhibit mutations in genes encoding subunits of the SWI/SNF chromatin remodeling complex, and ARID1A is the most frequently mutated gene among them (9).

Previous studies identified that mutations in ARID1A were not sufficient to induce gastric cancer; however, the concomitant activation of phosphatidylinositol-4,5-bisphosphate 3-kinase catalytic subunit $\alpha$ (PIK3CA) may promote gastric cancer development (10). PIK3CA is located on chromosome 3q26.3 and encodes a catalytic subunit of $110 \mathrm{kDa}$ (11). PIK3CA is mutated in various types of cancer, and $>80 \%$ of the mutations affecting PIK3CA are located in exons 9 and 20, including mutations that lead to the substitution of glutamic 
acid to lysine in position 542 (E542K), E545K and histidine to arginine in position 1047 (12). Cancer cells with PIK3CA mutations exhibit increased sensitivity to inhibitors of the phosphatidylinositol 3-kinase (PI3K)/AKT serine/threonine kinase (AKT) signaling pathway (13). Multiple growth factors affecting proliferation, protein translation, autophagy and metabolism are able to activate the PI3K/AKT pathway, and this pathway has been identified to be activated in $~ 30 \%$ of patients with gastric cancer (11).

Although the roles of ARID1A and PIK3CA in gastric cancer have been previously examined, our previous study (14), to the best of the authors' knowledge, was the first one to investigate the association among EBV, ARID1A and PIK3CA in gastric cancer. In the present study, the number of samples analyzed was increased compared with our previous investigation. In addition, the present study identified which mutations occurred in the coding sequences of PIK3CA and ARID1A in numerous tumor samples with the aim of investigating the association among EBV, ARID1A and PIK3CA at the genetic level.

\section{Materials and methods}

Samples and patients. All samples were collected from patients diagnosed with primary gastric cancer at The Central South University Xiangya School of Medicine Affiliated Haikou Hospital (Haikou, China) between January 2015 and November 2017. Consent was obtained prior to sample collection. Patients exhibiting primary gastric cancer were included in the study, whereas patients presenting metastatic gastric carcinoma were excluded. The Central South University Xiangya School of Medicine Affiliated Haikou Hospital Ethical Review Committee approved all the experiments performed on human tissues (permit no. 2015013). Among 300 patients with gastric cancer, 69\% (207/300) were male and $31 \%$ (93/300) were female. The mean age of the patients was $45 \pm 12.405$ years (range, 22-93 years). A total of 300 gastric cancer tissues were selected as the experimental group, whereas, 50 matched adjacent normal gastric tissues were selected as the control group. Adjacent normal tissue was defined as tissue within $3 \mathrm{~cm}$ from gastric cancer without the presence of cancer cells, following a microscope examination.

EBER in situ hybridization (ISH). All tissue samples were fixed in $10 \%$ neutral formalin solution using a tissue processor (cat. no. APS300S, Leica Microsystems GmbH, Wetzlar, Germany) for $9 \mathrm{~h}$ at room temperature, and subsequently dehydrated and embedded in paraffin at $65^{\circ} \mathrm{C}$ for $3 \mathrm{~h}$. All paraffin-embedded tissues were sectioned to a thickness of 4-6 $\mu \mathrm{m}$ and incubated in a $65^{\circ} \mathrm{C}$ thermostatic oven for 2 h. Subsequently, an EBER ISH kit was used (OriGene Technologies, Inc., Beijing, China), according to the manufacturer's protocol. Tissues were stained with 3,3-diaminobenzidine (DAB; 1:100) for 3-5 min at room temperature, and counterstained with hematoxylin for $2 \mathrm{~min}$ at room temperature. Subsequently, ethanol was used to dehydrate the sections at room temperature. Positive infected cells presented stained nuclei and unstained cytoplasm. The results of ISH were analyzed by two experienced pathologists using a light microscope (BX53; Olympus Corporation, Tokyo, Japan; magnification, x40, x100, x200 and x400).

Immunohistochemistry. All tissues were fixed in $10 \%$ neutral formalin solution using a tissue processor (cat. no. APS300S, Leica Microsystems $\mathrm{GmbH}$ ) for $9 \mathrm{~h}$ at room temperature, dehydrated and embedded in paraffin for $3 \mathrm{~h}$ at $65^{\circ} \mathrm{C}$. Subsequently, the paraffin-embedded tissues were sectioned to a thickness of 3-4 $\mu \mathrm{m}$ and incubated in a thermostatic oven at $65^{\circ} \mathrm{C}$ for $>2 \mathrm{~h}$. The sections were placed in xylene and in different concentrations of ethanol in order to rehydrate the tissues. The ethanol was subsequently removed by washing with distilled water for $10 \mathrm{~min}$, and the sections were incubated with hydrogen peroxide in the dark. Antigen retrieval was performed with EDTA (1:500, $\mathrm{pH}$ 8.0; OriGene Technologies, Inc.) using a pressure cooker. The tissue sections were heated at $100^{\circ} \mathrm{C}$ in EDTA solution and were boiled for $2.5 \mathrm{~min}$, the sections were subsequently cooled with cold water for $10 \mathrm{~min}$. The sections were washed three times with PBS. Following antigen retrieval, the samples were incubated overnight at $4^{\circ} \mathrm{C}$ with the following antibodies: ARID1A (1:800; cat. no. HPA005456; Sigma-Aldrich; Merck KGaA, Darmstadt, Germany), PIK3CA (1:300; cat. no. orb178194; Biorbyt Ltd., Cambridge, UK), Epstein-Barr nuclear antigen 1 (EBNA1; 1:10; cat. no. DP15L-100UGCN; Merck KGaA), EBV latent membrane protein 1 (LMP1; 1:1; cat. no. MAB-0063; Fuzhou Maixin Biotech. Co., Ltd., Fuzhou, China). Following a wash with PBS, the horseradish peroxidase-labeled secondary antibody was added (1:1; cat. no. PV-6000D; OriGene Technologies, Inc.) to the tissues and incubated at $37^{\circ} \mathrm{C}$ for $30 \mathrm{~min}$. DAB was used as the detection reagent for $5 \mathrm{~min}$, the samples were counterstained with hematoxylin for 2 min at room temperature, and subsequently dehydrated in a gradient series of ethanol. The samples were considered to be ARID1A-positive if the nuclei of tumor cells were stained and PIK3CA-positive if the cytoplasm was stained by DAB. The results were analyzed by two experienced pathologists with a light microscope (BX53; Olympus Corporation; magnification, $\mathrm{x} 40, \mathrm{x} 100, \mathrm{x} 200$ and $\mathrm{x} 400$ ). A score was subsequently assigned to the samples according to the number of positive cells ( 0 , no cells stained; $1,1-30 \%$ cells stained; $2,31-70 \%$ cells stained; 3, 71-100\% cells stained) and to the intensity of the signal ( 0 , no signal; 1 , mild signal; 2 , moderate signal; 3 , strong signal). The final score was calculated by multiplying the two scores. The samples were further categorized based on the final score, which was defined as '-' if the final score was 0 , ' + ' for a score of 1-3, ' ++ ' for 4-6 and ' +++ ' for 7-9.

Microscope and image analysis. Image-Pro Plus software (version 6.0; Media Cybernetics, Inc., Rockville, MD, USA) was used to convert the immunohistochemistry results to semiquantitative data. A total of ten microscopic fields were randomly selected on each slide, and the stromal elements were removed. Subsequently, the average absorbance was determined based on the area and intensity of positively stained samples. When significant differences were detected in the staining results among distinct fields of view within one section, the integral absorbance was determined as the integral of the background average grey value divided by the determinant objective average grey value (15). The absorbance 
Table I. Fluorescence polymerase chain reaction results.

\begin{tabular}{|c|c|c|c|c|c|c|}
\hline Mutation type & $\mathrm{Ct}$ value & H1047R & H1047L & E542K & E545K & E545D \\
\hline Strongly positive & $\mathrm{Ct}$ value of mutation & $\mathrm{Ct}<25$ & $\mathrm{Ct}<26$ & $\mathrm{Ct}<25$ & $\mathrm{Ct}<26$ & $\mathrm{Ct}<26$ \\
\hline \multirow[t]{2}{*}{ Weakly positive } & $\mathrm{Ct}$ value of mutation & $25 \leq \mathrm{Ct}$ & $26 \leq \mathrm{Ct}$ & $25 \leq \mathrm{Ct}$ & $26 \leq \mathrm{Ct}$ & $26 \leq \mathrm{Ct}$ \\
\hline & $\Delta \mathrm{Ct}$ cut-off & 11 & 12 & 12 & 12 & 12 \\
\hline Negative & $\mathrm{Ct}$ value of mutation & $\mathrm{Ct}>28$ & $\mathrm{Ct} \geq 29$ & $\mathrm{Ct} \geq 29$ & $\mathrm{Ct} \geq 29$ & $\mathrm{Ct} \geq 29$ \\
\hline
\end{tabular}

Ct, cycle threshold; H, histidine; R, arginine; L, leucine; E, glutamic acid; K, lysine; D, aspartic acid.

averages were calculated and the data were analyzed in order to measure the immunohistochemical intensity in a semiquantitative way.

Fluorescence polymerase chain reaction (PCR). A total of 28 EBV-positive samples, identified by EBER ISH, 30 gastric cancer tissues and 10 adjacent normal tissues were selected. Gastric cancer tissues (samples with low ARID1A expression and high PIK3CA expression) and normal tissues (samples with high ARID1A expression and low PIK3CA expression) were selected based on the immunohistochemical results. The DNA from the selected samples was extracted, and the PIK3CA Five Mutations Detection kit (Amoy Diagnostics Co., Ltd., Xiamen, China) was used, according to the manufacturer's protocol. The fluorophore and the DNA polymerase were included in the mutation detection kit. The following primers were used for the detection of mutations in PIK3CA: PIK3CA-9 forward, 5'-TAT GTCTTAGATTGGTTCTTTCCTG-3' and reverse, 5'-AGC ATTTAATGTGCCAACTACCA-3'; PIK3CA-20 forward, 5'-TTTCTCAATGATGCTTGGCTCT-3' and reverse, 5'-TGT TCTTGCTGTAAATTCTAATGCT-3'. The LightCycler 480 (Roche Diagnostics, Basel, Switzerland) thermocycler was used. The thermocycling conditions were the following: Initial denaturation at $95^{\circ} \mathrm{C}$ for $5 \mathrm{~min}$ followed by 15 cycles of $95^{\circ} \mathrm{C}$ for $25 \mathrm{sec}, 64^{\circ} \mathrm{C}$ for $20 \mathrm{sec}$ and $72^{\circ} \mathrm{C}$ for 20 , and by 31 subsequent cycles of $93^{\circ} \mathrm{C}$ for $25 \mathrm{sec}, 60^{\circ} \mathrm{C}$ for $35 \mathrm{sec}$ and $72^{\circ} \mathrm{C}$ for $20 \mathrm{sec}$. The $\mathrm{Ct}$ value and the external control $\mathrm{Ct}$ values were determined for each sample. According to the $\mathrm{Ct}$ values, the results were divided into negative, mildly positive and strongly positive. The association between $\mathrm{Ct}$ values and mutations are presented in Table I.

TaqMan ${ }^{\circledR}$ single nucleotide polymorphism (SNP) genotyping assays. The genetic mutations in ARID1A and PIK3CA were examined by TaqMan ${ }^{\circledR}$ SNP genotyping assays (Sangon Biotech Co., Ltd., Shanghai, China). To confirm the results of fluorescence PCR, the PIK3CA mutations were investigated by SNP genotyping assays. The following primers (5' to $3^{\prime}$ direction) were used for the TaqMan ${ }^{\circledR}$ SNP genotyping assay: ARID1A-7 forward, CATGCAAGTGGCTGCTAAAGA and reverse, GGA TTAAAGGCAAAGGACAGC; ARID1A-11 forward, GCA CAGCACCCAGTACATAACAT and reverse, CTTATCTTC TGGGAAACCTGTGC; ARID1A-15 forward GGCTTCACC TTGTCATCCCTTA and reverse, AAGGCATGAGATTCG GCTGT; ARID1A-20-1 forward, GGTAGGTTGGGCAGA AGAAAGA and reverse, CTTATCTGAGCAGTCCACCA CAA; ARID1A-20-2 forward, TAGTTGAAAATGATGAGG
AGATAGC and reverse, CCACCACCACTCCACTTTGTT; ARID1A-20-3 forward, GAGGACGAACCCCACAGTAAG and reverse, CAGCAGTACCACAGCCATCTC; ARID1A-20-4 forward, AACTCAGCATCCAGGACAACAAT and reverse, ACAAGGAACAGAAAGGCGTGA; PIK3CA-9 forward, TAT GTCTTAGATTGGTTCTTTCCTG and reverse, AGCATT TAATGTGCCAACTACCA; PIK3CA-20 forward, TTTCTC AATGATGCTTGGCTCT and reverse, TGTTCTTGCTGT AAATTCTAATGCT. The thermocycling conditions were the following: Initial denaturation at $94^{\circ} \mathrm{C}$ for $3 \mathrm{~min}$, followed by 35 cycles of $94^{\circ} \mathrm{C}$ for $30 \mathrm{sec}, 58^{\circ} \mathrm{C}$ for $30 \mathrm{sec}$ and $72^{\circ} \mathrm{C}$ for 30-60 sec. The last extension was performed at $72^{\circ} \mathrm{C}$ for $10 \mathrm{~min}$. The 68 DNA samples analyzed by fluorescence PCR were tested by Sangon Biotech Co., Ltd. in order to detect the genetic mutation present in the exons that were identified to be frequently altered in the two genes (exons 7, 11, 15 and 20 of ARID1A; exons 9 and 20 of PIK3CA) $(12,16)$.

Statistical analysis. SPSS (version 23; IBM Corp., Armonk, NY, USA) was used to analyze the data. The significant association between ARID1A and PIK3CA and among the clinicopathological features of patients was assessed using the $\chi^{2}$ test. Spearman's correlation analysis was used to examine the correlation between the expression levels of the two genes in various groups. $\mathrm{P}<0.05$ was considered to indicate a statistically significant difference.

\section{Results}

Clinicopathological features. Among 300 patients with gastric cancer, 69\% (207/300) were male and 31\% (93/300) were female. The patients had a mean age of $45 \pm 12.405$ years (range, 22-93 years). The group of patients whose ages ranged between 61 and 70 years exhibited the highest occurrence rate $(31.0 \%)$. In total, $74 \%$ (222/300) of patients with gastric cancer presented tubular adenocarcinoma, 69.7\% (209/300) exhibited poorly differentiated gastric cancer, 67\% (201/300) presented lymphatic metastasis at the initial diagnosis, and in $75 \%(225 / 300)$ of the patients, cancer cells invaded the serosal layer or adjacent organs and tissues.

ISH analysis. ISH was used to examine the EBV infection status of all 350 samples (300 samples of gastric cancer and 50 adjacent normal tissues). In total, 9.3\% (28/300) of gastric cancer tissues were identified as EBV-positive (Fig. 1A), whereas, no EBV-positive cells were identified in adjacent normal gastric mucosa (Fig. 1B). 
Table II. Differences in the expression levels of ARID1A and PIK3CA between GC and normal gastric tissues.

A, ARID1A expression

\begin{tabular}{|c|c|c|c|c|c|c|}
\hline Type of tissue & $\mathrm{n}$ & - & + & ++ & +++ & P-value \\
\hline Normal tissue & 50 & 0 & 19 & 27 & 4 & $<0.0001^{\mathrm{a}}$ \\
\hline GC tissue & 300 & 34 & 178 & 82 & 6 & \\
\hline
\end{tabular}

\section{B, PIK3CA expression}

\begin{tabular}{|c|c|c|c|c|c|c|}
\hline Type of tissue & $\mathrm{n}$ & - & + & ++ & +++ & P-value \\
\hline Normal tissue & 50 & 0 & 2 & 35 & 13 & $<0.0001^{\mathrm{a}}$ \\
\hline GC tissue & 300 & 0 & 0 & 118 & 182 & \\
\hline
\end{tabular}

ARID1A, AT-rich interaction domain 1A; PIK3CA, phosphatidylinositol-4,5-bisphosphate 3-kinase catalytic subunit $\alpha$; -, negative; +, weakly positive; ++, moderately positive; +++, strongly positive; GC, gastric cancer. ${ }^{\mathrm{a}} \mathrm{P}<0.05$ vs. respective control.
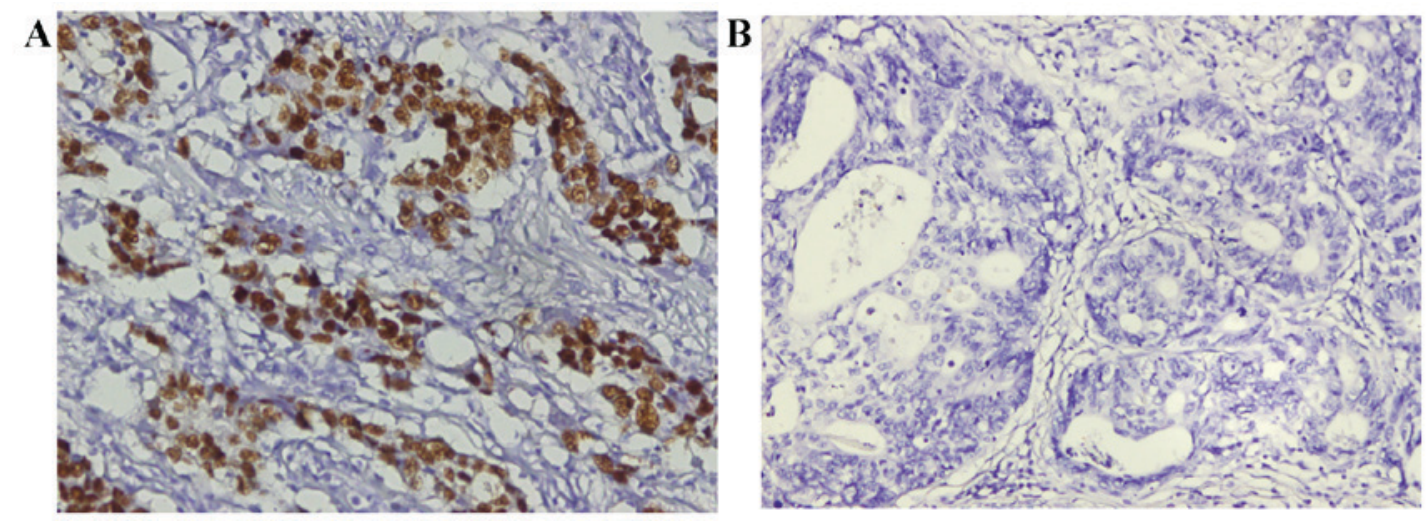

Figure 1. ISH against EBER. (A) EBER-positive gastric cancer tissue detected by ISH. (B) EBER-negative gastric cancer tissue detected by ISH. EBER, Epstein-Barr virus encoded small RNA; ISH, in situ hybridization.

A

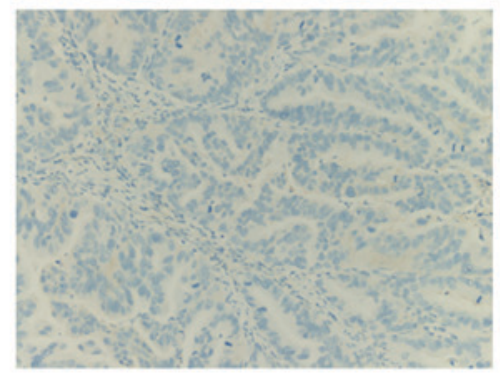

D

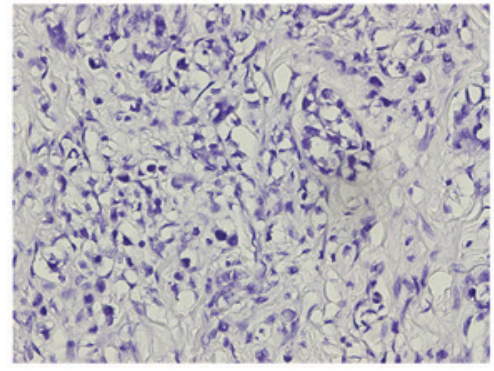

B

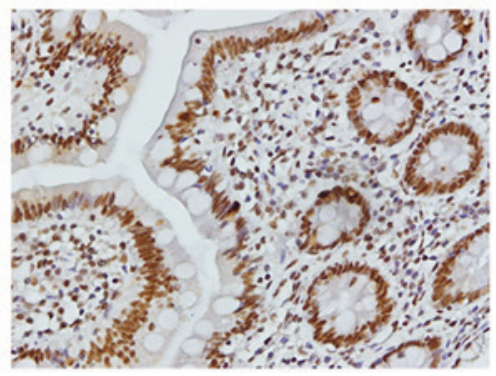

$\mathbf{E}$

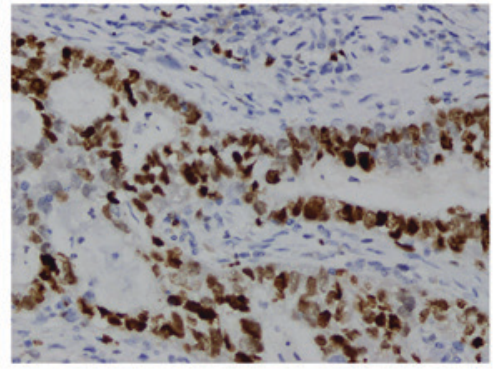

C

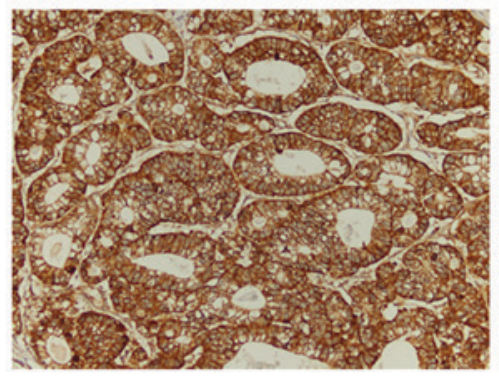

$\mathbf{F}$

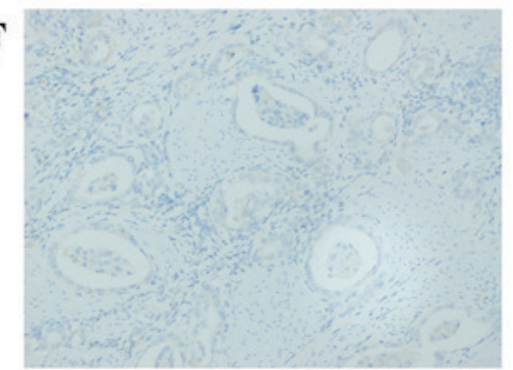

Figure 2. Immunohistochemistry results. (A) ARID1 A-negative gastric cancer tissue. (B) ARID1A-positive gastric cancer tissue. (C) PIK3CA-positive gastric cancer tissue. (D) EBNA1-negative EBVaGC sample. (E) EBNA1-positive EBVaGC sample. (F) EBV latent membrane protein 1-negative EBVaGC sample. EBV, Epstein-Barr virus; ARID1A, AT-rich interaction domain 1A; PIK3CA, phosphatidylinositol-4,5-bisphosphate 3-kinase catalytic subunit $\alpha$; EBNA1, Epstein-Barr nuclear antigen 1; EBVaGC, EBV-associated gastric cancer. 
Table III. Differences in PIK3CA and ARID1A expression between gastric cancer and normal tissues.

\begin{tabular}{lccc}
\hline Groups & Cases & Mean density (ARID1A) & Mean density (PIK3CA) \\
\hline Gastric cancer & 300 & $0.1649 \pm 0.0043$ & $0.4187 \pm 0.0098$ \\
Normal tissue & 50 & $0.2143 \pm 0.0094$ & $0.2649 \pm 0.0062$ \\
\hline
\end{tabular}

ARID1A, AT-rich interaction domain 1A; PIK3CA, phosphatidylinositol-4,5-bisphosphate 3-kinase catalytic subunit $\alpha$.

Table IV. Differences in the expression levels of ARID1A and PIK3CA between EBV negative gastric cancer and EBV positive gastric cancer tissues.

A, ARID1A expression

\begin{tabular}{lrrrrr}
\hline Type of gastric cancer tissue & $\mathrm{n}$ & - & + & ++ & +++ \\
\hline EBV negative & 272 & 28 & 164 & 75 & 5 \\
EBV positive & 28 & 6 & 14 & 7 & 1 \\
\hline
\end{tabular}

\section{B, PIK3CA expression}

\begin{tabular}{lrrrrr}
\hline Type of gastric cancer tissue & $\mathrm{n}$ & - & + & ++ & +++ \\
\hline EBV negative & 272 & 0 & 0 & 110 & 162 \\
EBV positive & 28 & 0 & 0 & 8 & 20
\end{tabular}

ARID1A, AT-rich interaction domain 1A; PIK3CA, phosphatidylinositol-4,5-bisphosphate 3-kinase catalytic subunit $\alpha$; -, negative; +, weakly positive; ++, moderately positive; +++, strongly positive; EBV, Epstein-Barr virus.

Table V. Difference in the expression levels of PIK3CA and ARID1A between EBVaGC and EBVnGC.

\begin{tabular}{lrrr}
\hline Type of gastric cancer tissue & Cases & Mean density (ARID1A) & Mean density (PIK3CA) \\
\hline EBVnGC & 272 & $0.1664 \pm 0.0045$ & $0.4167 \pm 0.0102$ \\
EBVaGC & 28 & $0.1502 \pm 0.0156$ & $0.4393 \pm 0.0333$ \\
\hline
\end{tabular}

ARID1A, AT-rich interaction domain 1A; PIK3CA, phosphatidylinositol-4,5-bisphosphate 3-kinase catalytic subunit $\alpha$; EBV, Epstein-Barr virus; EBVaGC EBV-associated gastric cancer; EBVnGC, EBV-negative gastric cancer.

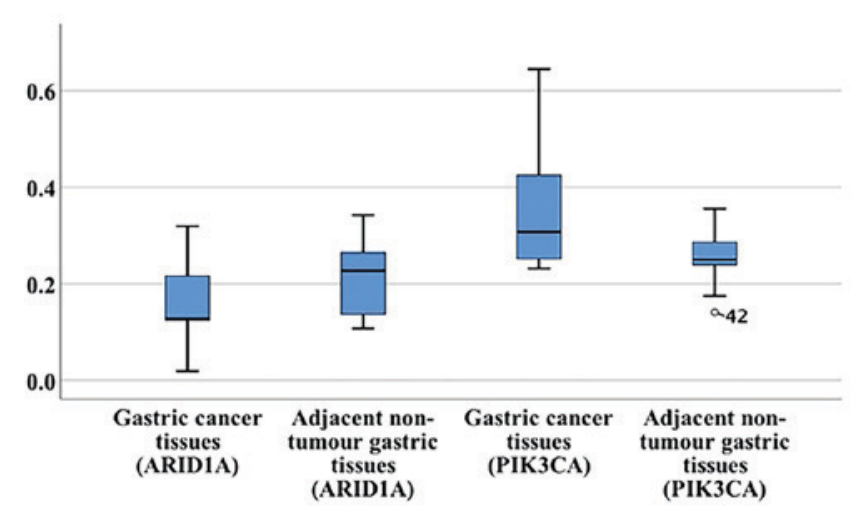

Figure 3. Box plot indicating the protein expression levels of ARID1A and PIK3CA in gastric cancer tissues and normal gastric tissues measured by microscopy and image analysis. ARID1A, AT-rich interaction domain 1A; PIK3CA, phosphatidylinositol-4,5-bisphosphate 3-kinase catalytic subunit $\alpha$.

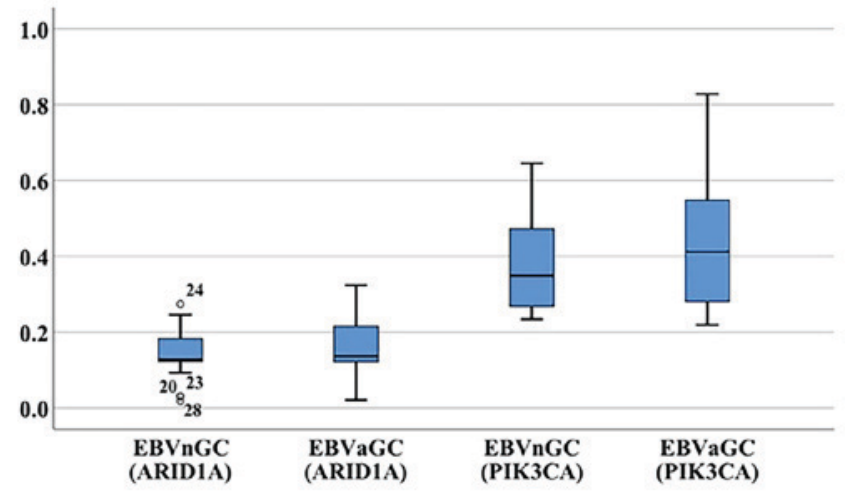

Figure 4. Box plot indicating the protein expression levels of ARID1A and PIK3CA in EBVaGC tissues and EBVnGC tissues measured by microscopy and image analysis. ARID1A, AT-rich interaction domain 1A; PIK3CA, phosphatidylinositol-4,5-bisphosphate 3-kinase catalytic subunit $\alpha$; EBV, Epstein-Barr virus; EBVaGC EBV-associated gastric cancer; EBVnGC, EBV-negative gastric cancer. 
Table VI. Correlations of clinicopathological parameters and the protein expression levels of ARID1A and PIK3CA in patients with gastric cancer.

\begin{tabular}{|c|c|c|c|c|c|c|}
\hline Clinicopathological features & Total $(n=300)$ & - & + & ++ & +++ & P-value \\
\hline Age & & & & & & 0.809 \\
\hline$\leq 60$ & 147 & 16 & 90 & 37 & 4 & \\
\hline$>60$ & 153 & 18 & 88 & 45 & 2 & \\
\hline Sex & & & & & & 0.247 \\
\hline Male & 207 & 22 & 120 & 61 & 4 & \\
\hline Female & 93 & 12 & 58 & 21 & 2 & \\
\hline Differentiation & & & & & & 0.343 \\
\hline High & 91 & 8 & 62 & 21 & 0 & \\
\hline Low & 209 & 26 & 116 & 61 & 6 & \\
\hline Histological classification & & & & & & 0.345 \\
\hline Tubular adenocarcinoma & 222 & 25 & 128 & 64 & 5 & \\
\hline Other type & 78 & 9 & 50 & 18 & 1 & \\
\hline Lymphatic metastasis & & & & & & 0.528 \\
\hline Yes & 201 & 22 & 118 & 56 & 5 & \\
\hline No & 99 & 12 & 60 & 26 & 1 & \\
\hline Infiltration & & & & & & $0.040^{\mathrm{a}}$ \\
\hline Mucosa and muscular layer & 75 & 12 & 47 & 15 & 1 & \\
\hline Serosa & 225 & 22 & 131 & 67 & 5 & \\
\hline
\end{tabular}

B, PIK3CA expression

\begin{tabular}{|c|c|c|c|c|c|c|}
\hline Clinicopathological features & Total $(n=300)$ & - & + & ++ & +++ & P-value \\
\hline Age & & & & & & 0.966 \\
\hline$\leq 60$ & 147 & 0 & 0 & 58 & 89 & \\
\hline$>60$ & 153 & 0 & 0 & 60 & 93 & \\
\hline Sex & & & & & & 0.882 \\
\hline Male & 207 & 0 & 0 & 82 & 125 & \\
\hline Female & 93 & 0 & 0 & 36 & 57 & \\
\hline Differentiation & & & & & & $0.009^{\mathrm{a}}$ \\
\hline High & 91 & 0 & 0 & 46 & 45 & \\
\hline Low & 209 & 0 & 0 & 72 & 137 & \\
\hline Histological classification & & & & & & 0.322 \\
\hline Tubular adenocarcinoma & 222 & 0 & 0 & 91 & 131 & \\
\hline Other type & 78 & 0 & 0 & 27 & 51 & \\
\hline Lymphatic metastasis & & & & & & $<0.001^{\mathrm{a}}$ \\
\hline Yes & 201 & 0 & 0 & 59 & 142 & \\
\hline No & 99 & 0 & 0 & 59 & 40 & \\
\hline Infiltration & & & & & & $<0.001^{\mathrm{a}}$ \\
\hline Mucosa and muscular layer & 75 & 0 & 0 & 72 & 3 & \\
\hline Serosa & & 0 & 0 & 46 & 179 & \\
\hline
\end{tabular}

ARID1A, AT-rich interaction domain 1A; PIK3CA, phosphatidylinositol-4,5-bisphosphate 3-kinase catalytic subunit $\alpha$; -, negative; +, weakly positive; ++, moderately positive; +++, strongly positive. ${ }^{a} \mathrm{P}<0.05$ vs. respective control.

Immunohistochemistry. Immunohistochemistry was used to investigate ARID1A and PIK3CA protein expression levels in gastric cancer and adjacent normal tissues. In order to test the $\mathrm{EBV}$ infection state in EBVaGC, the protein expression levels of 


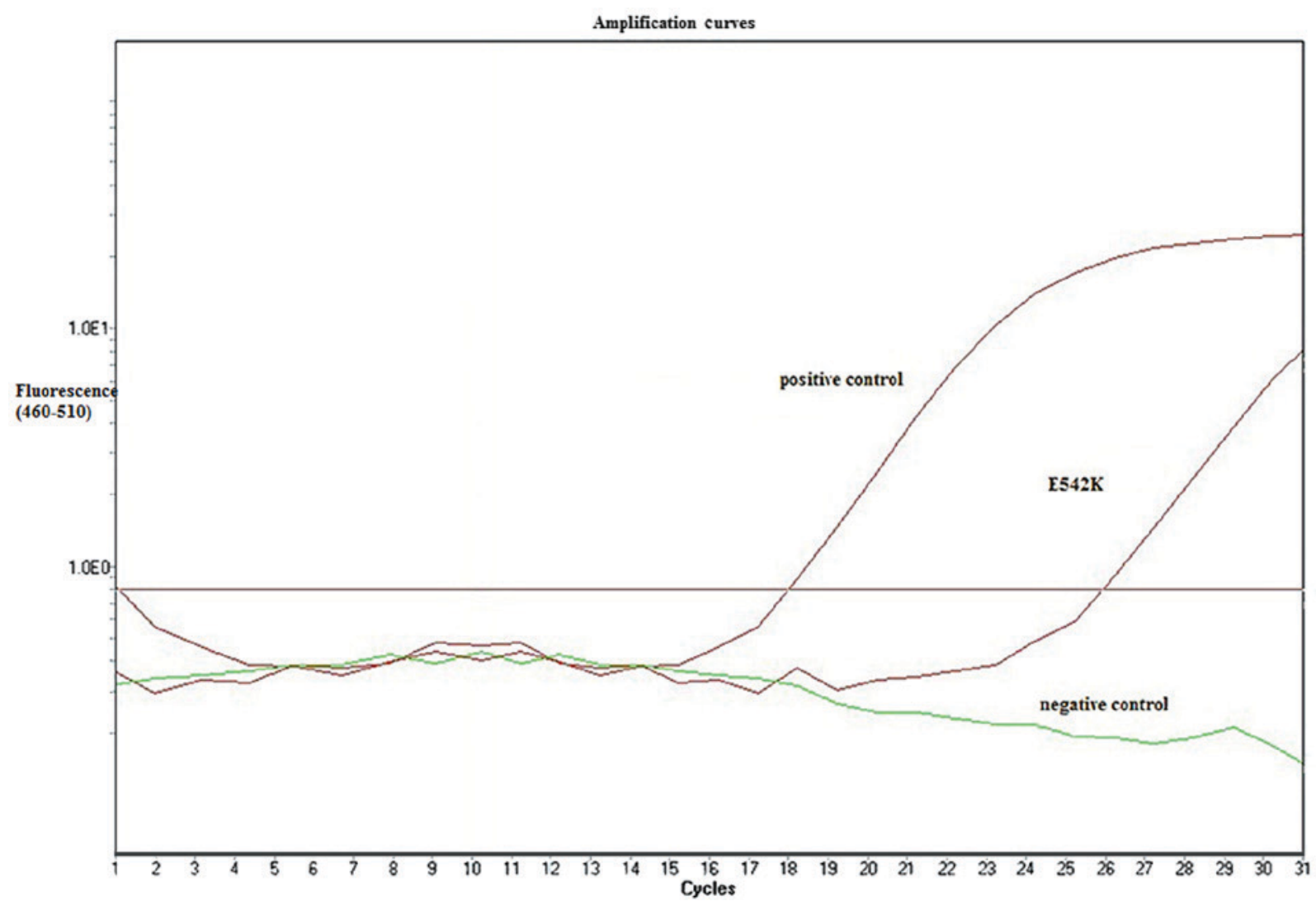

Figure 5. Fluorescence PCR. The PIK3CA mutation leading to E542K was detected using fluorescence PCR. PCR, polymerase chain reaction; E542K, glutamic acid to lysine substitution in position 542. PIK3CA, phosphatidylinositol-4,5-bisphosphate 3-kinase catalytic subunit $\alpha$.

Table VII. Correlation analysis of the protein expression levels of ARID1A and PIK3CA in gastric cancer tissues.

\begin{tabular}{lrrrrr}
\hline & \multicolumn{4}{c}{ ARID1A expression } \\
\cline { 2 - 5 } PIK3CA expression & - & + & ++ & +++ & $\mathrm{n}$ \\
\hline- & 0 & 0 & 0 & 0 & 0 \\
+ & 0 & 0 & 0 & 0 & 0 \\
++ & 15 & 54 & 43 & 6 & 118 \\
+++ & 19 & 124 & 39 & 0 & 182 \\
$\mathrm{n}$ & 34 & 178 & 82 & 6 & 300 \\
\hline
\end{tabular}

$\mathrm{r}=-0.167, \mathrm{P}=0.004$. ARID1A, AT-rich interaction domain $1 \mathrm{~A}$; PIK3CA, phosphatidylinositol-4,5-bisphosphate 3-kinase catalytic subunit $\alpha ;-$, negative; + , weakly positive; ++ , moderately positive; +++ , strongly positive.

EBNA1 and LMP1 were examined via immunohistochemistry. In total, 27 out of 28 EBVaGC samples were EBNA1-positive, whereas no EBVaGC sample was LMP1-positive (Fig. 2). In order to convert the immunohistochemistry results to semiquantitative data, Image-Pro Plus software was used. The box plots suggested that the expression level of ARID1A was downregulated in gastric cancer tissues compared with adjacent normal tissues, whereas the expression level of PIK3CA was upregulated in gastric cancer (Fig. 3). However, the expression
Table VIII. Correlation analysis of the protein expression levels of ARID1A and PIK3CA in Epstein-Barr virus-positive gastric cancer.

\begin{tabular}{lrrrrr}
\hline & \multicolumn{4}{c}{ ARID1A expression } \\
\cline { 2 - 5 } PIK3CA expression & - & + & ++ & +++ & $n$ \\
\hline- & 0 & 0 & 0 & 0 & 0 \\
+ & 0 & 0 & 0 & 0 & 0 \\
++ & 1 & 2 & 4 & 1 & 8 \\
+++ & 5 & 12 & 3 & 0 & 20 \\
$\mathrm{n}$ & 6 & 14 & 7 & 1 & 28 \\
\hline
\end{tabular}

$\mathrm{r}=-0.409, \mathrm{P}=0.031$. ARID1A, AT-rich interaction domain 1A; PIK3CA, phosphatidylinositol-4,5-bisphosphate 3-kinase catalytic subunit $\alpha ;-$, negative; + , weakly positive; ++ , moderately positive; +++ , strongly positive.

levels of ARID1A and PIK3CA were similar between EBVaGC and EBVnGC (Fig. 4). The difference in the protein expression levels of ARID1A and PIK3CA between gastric cancer tissues and adjacent normal gastric tissues was statistically significant (Tables II and III). The difference in the protein expression levels of ARID1A and PIK3CA between EBV-negative gastric cancer (EBVnGC) and EBVaGC was not significant (Tables IV and V). The expression level of PIK3CA was identified to be associated with differentiation, depth of tumor invasion and lymphatic 

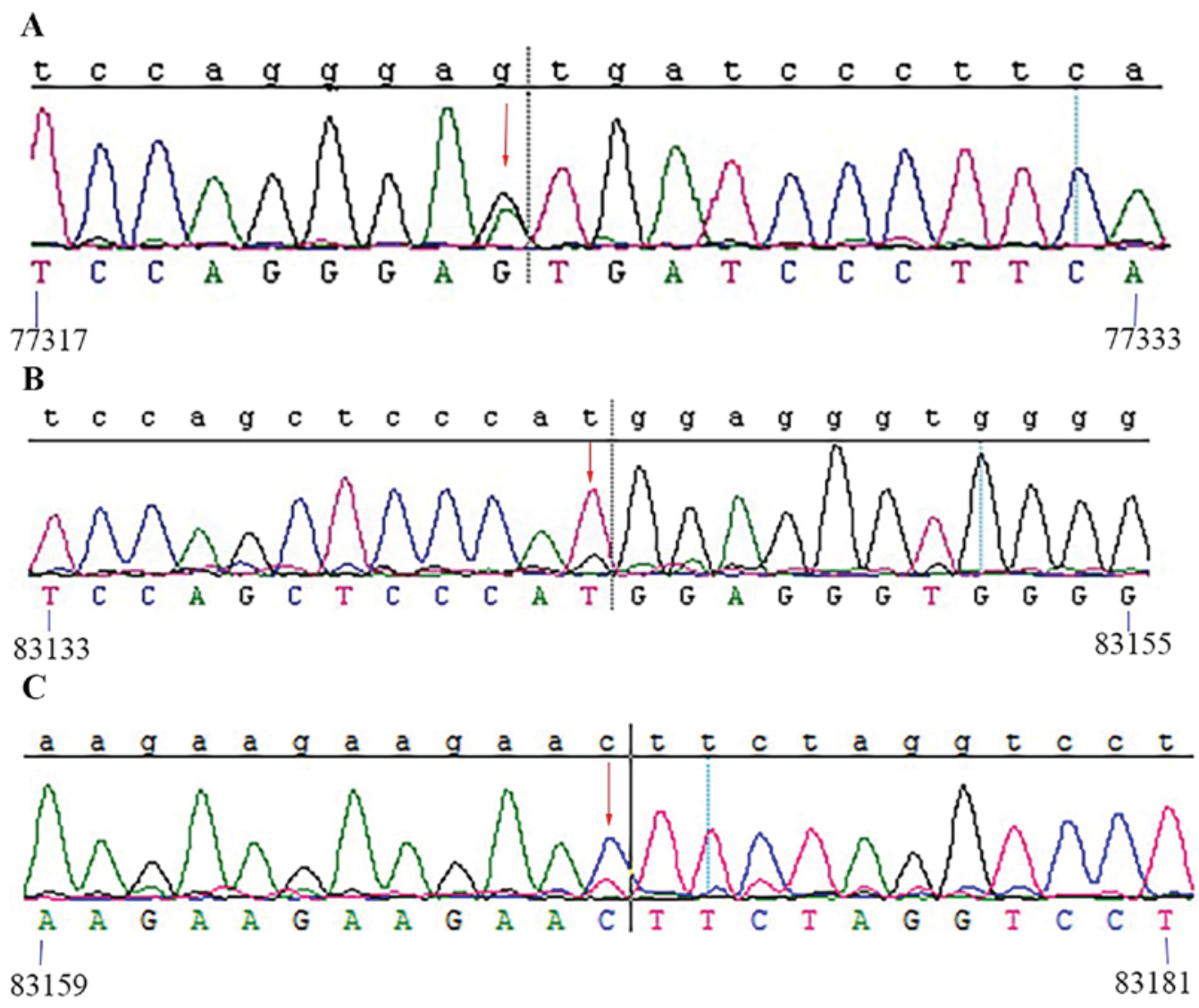

D

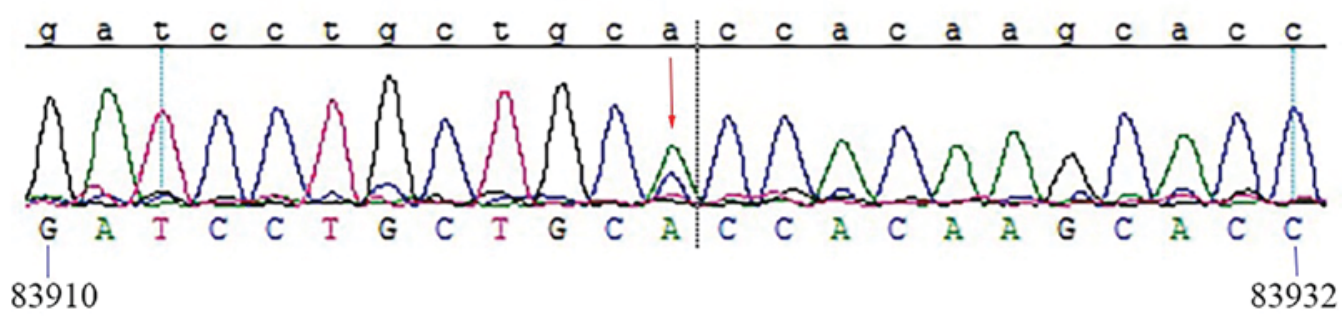

E

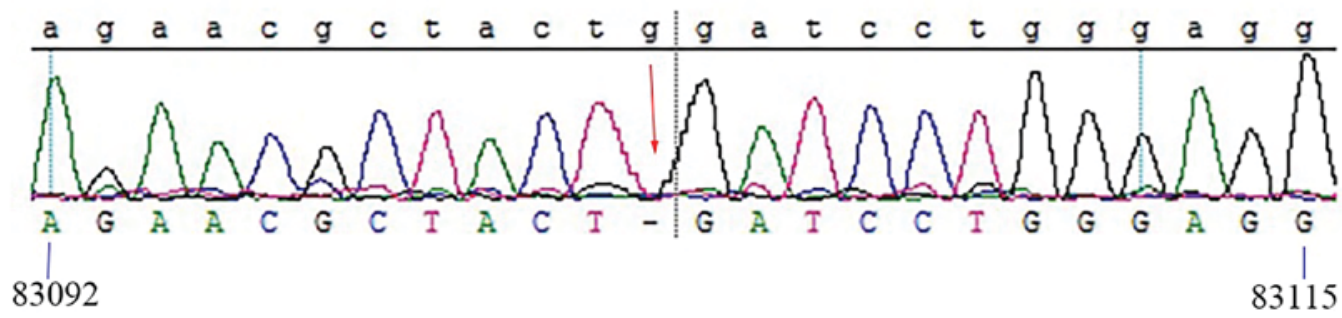

Figure 6. Genotyping results for ARID1A gene. (A) A to G mutation. (B) G to T mutation. (C) C to T. (D) A to C mutation. (E) Deletion mutation. A, adenine; $\mathrm{G}$, guanine; C, cytosine; T, thymine; ARID1A, AT-rich interaction domain 1A.

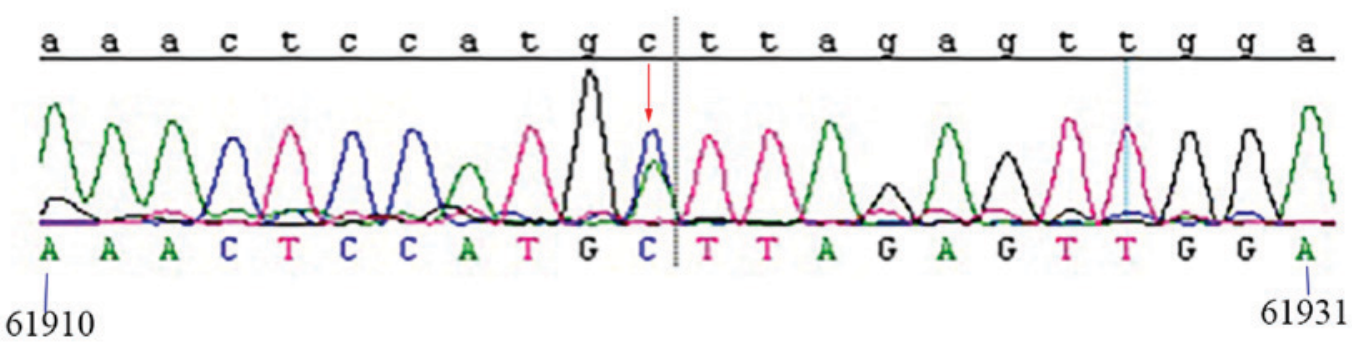

Figure 7. Genotyping results for PIK3CA gene. A to G mutation leading to the substitution of a glutamic acid in position 542 with a lysine. A, adenine; $\mathrm{G}$, guanine; C, cytosine; T, thymine. PIK3CA, phosphatidylinositol-4,5-bisphosphate 3-kinase catalytic subunit $\alpha$. 
Table IX. Genetic mutations and protein alterations in patients with gastric cancer exhibiting mutations in ARID1A and/or PIK3CA.

\begin{tabular}{|c|c|c|c|c|c|c|}
\hline $\begin{array}{l}\text { Tissue } \\
\text { number }\end{array}$ & $\begin{array}{l}\text { Immunostaining } \\
\text { (ARID1A) }\end{array}$ & $\begin{array}{l}\text { ARID1A } \\
\text { mutation }\end{array}$ & $\begin{array}{l}\text { Amino } \\
\text { acid }\end{array}$ & $\begin{array}{l}\text { Immunostaining } \\
\text { (PIK3CA) }\end{array}$ & $\begin{array}{l}\text { PIK3CA } \\
\text { mutation }\end{array}$ & $\begin{array}{l}\text { Amino } \\
\text { acid }\end{array}$ \\
\hline 18 & + & $\mathrm{A} / \mathrm{G}$ & Ser $\rightarrow$ Asn & +++ & & \\
\hline 32 & - & $\mathrm{A} / \mathrm{G}$ & Gly $\rightarrow$ Asp & +++ & $\mathrm{A} / \mathrm{G}$ & Glu $\rightarrow$ Lys \\
\hline 45 & - & $\mathrm{A} / \mathrm{G}$ & Gly $\rightarrow$ Asp & +++ & $\mathrm{A} / \mathrm{G}$ & Glu $\rightarrow$ Lys \\
\hline 48 & - & $\mathrm{A} / \mathrm{G}$ & Arg $\rightarrow$ Lys & +++ & & \\
\hline 67 & + & $\mathrm{A} / \mathrm{G}$ & Glu $\rightarrow$ Lys & +++ & $\mathrm{A} / \mathrm{G}$ & Glu $\rightarrow$ Lys \\
\hline 96 & - & $\mathrm{A} / \mathrm{G}$ & $\mathrm{Ile} \rightarrow \mathrm{Val}$ & +++ & & \\
\hline 118 & + & $\mathrm{C} / \mathrm{T}$ & $\mathrm{Ile} \rightarrow$ Pro & +++ & $\mathrm{A} / \mathrm{G}$ & Glu $\rightarrow$ Lys \\
\hline 135 & - & $\mathrm{C} / \mathrm{T}$ & Ala $\rightarrow$ Val & +++ & $\mathrm{A} / \mathrm{G}$ & Glu $\rightarrow$ Lys \\
\hline 143 & - & $-/ G$ & Frameshift & +++ & & \\
\hline 156 & - & $\mathrm{A} / \mathrm{G}$ & Gly $\rightarrow$ Asp & +++ & $\mathrm{A} / \mathrm{G}$ & Glu $\rightarrow$ Lys \\
\hline 166 & - & $\mathrm{A} / \mathrm{G}$ & $\operatorname{Arg} \rightarrow$ Lys & +++ & $\mathrm{A} / \mathrm{G}$ & Glu $\rightarrow$ Lys \\
\hline 193 & - & $-/ \mathrm{G}$ & Frameshift & +++ & & \\
\hline 204 & - & $\mathrm{C} / \mathrm{T}$ & Ile $\rightarrow$ Pro & +++ & $\mathrm{A} / \mathrm{G}$ & Glu $\rightarrow$ Lys \\
\hline 223 & - & $\mathrm{A} / \mathrm{G}$ & Arg $\rightarrow$ Lys & +++ & & \\
\hline 235 & - & $\mathrm{C} / \mathrm{T}$ & Ile $\rightarrow$ Pro & +++ & & \\
\hline 242 & - & $\mathrm{A} / \mathrm{G}$ & Ser $\rightarrow$ Asn & +++ & & \\
\hline 259 & - & $\mathrm{A} / \mathrm{G}$ & Gly $\rightarrow$ Asp & +++ & & \\
\hline $35 \mathrm{~N}$ & ++ & $\mathrm{C} / \mathrm{T}$ & $\mathrm{Ala} \rightarrow \mathrm{Val}$ & ++ & & \\
\hline $48 \mathrm{~N}$ & ++ & $\mathrm{A} / \mathrm{G}$ & Gly $\rightarrow$ Asp & ++ & & \\
\hline $56 \mathrm{~N}$ & ++ & $\mathrm{A} / \mathrm{G}$ & Gly $\rightarrow$ Asp & ++ & & \\
\hline
\end{tabular}

ARID1A, AT-rich interaction domain 1A; PIK3CA, phosphatidylinositol-4,5-bisphosphate 3-kinase catalytic subunit $\alpha$; -, negative; +, weakly positive; ++, moderately positive; +++, strongly positive; A, adenine; G, guanine; C, cytosine; T, thymine; Ala, alanine; Arg, arginine; Asn, asparagine; Asp, aspartic acid; Asn, asparagine; Glu, glutamic acid; Gly, glycine; Ile, isoleucine; Lys, lysine; Pro, proline; Ser, serine; Val, valine.

metastasis; however, no additional characteristics were identified to be associated with PIK3CA, including age, sex or histological type. The protein expression level of ARID1A was identified to be significantly associated with depth of tumor invasion, whereas, no association with additional clinicopathological characteristics was identified (Table VI). Correlation analysis suggested that the protein expression levels of ARID1A and PIK3CA were negatively correlated in gastric cancer $(r=-0.167$; Table VII). Specifically, the protein expression levels of ARID1A and PIK3CA were negatively correlated in EBVaGC ( $\mathrm{r}=-0.409$; Table VIII).

Fluorescence PCR. The PIK3CA Five Mutations Detection kit was used to test 58 gastric cancer tissues, $28 \mathrm{EBVaGC}$ and $30 \mathrm{EBVnGC}$, and 10 adjacent normal tissues. In total, eight EBVaGC samples (13.8\%) were identified to contain the point mutation leading to E542K (Fig. 5), whereas, no mutation was detected in adjacent normal tissues or EBVnGC.

TaqMan $^{\circledR}$ SNP genotyping assays. A total of 58 gastric cancer tissues, $28 \mathrm{EBVaGC}$ and $30 \mathrm{EBVnGC}$, and 10 adjacent normal tissues were sequenced. ARID1A was identified to be mutated in $62.1 \%(36 / 58)$ of gastric cancer samples, and $30.6 \%$ (11/36) were EBVaGC samples (Table IX). Genetic mutations of ARID1A were identified on exon 15 and 20, and the most common substitutions included adenine (A) to guanine $(\mathrm{G}), \mathrm{G}$ to thymine $(\mathrm{T})$, cytosine $(\mathrm{C})$ to $\mathrm{T}, \mathrm{A}$ to $\mathrm{C}$. Additionally, deletion mutations were identified (Fig. 6). Only eight mutations, A to C, were identified on exon 9 of PIK3CA (Fig. 7).

\section{Discussion}

EBV is a $\gamma$-herpesvirus, and EBV infection may promote lymphoproliferative disease, B cell lymphoma and natural killer T cell lymphoma (17). In total, $\sim 90 \%$ of the population has latent EBV infection (18). In our previous study, $11.63 \%$ of the tissues analyzed was EBV-positive; however, EBV was identified to be associated with $9.3 \%$ (28/300) of gastric cancer samples (14), in line with previous findings (19). In addition, EBV infection was identified more frequently in gastric cancer tissues compared with adjacent normal tissues, suggesting that EBV infection may be associated with the emergence of gastric cancer.

Previous studies demonstrated that the loss of ARID1A function in gastric cancer was correlated with malignancy and poor prognosis, thus resulting in a shortened survival and increased risk of recurrence $(20,21)$. Nevertheless, a previous study observed that a decrease in the expression level of ARID1A was associated with better prognosis (16). In the present study, it was identified that $11.3 \%(34 / 300)$ 
of gastric cancer tissues were positive for ARID1A, which was an increased number compared with our previous study $(9.30 \%)$ (14). Compared with adjacent normal tissues, the protein expression level of ARID1A in gastric cancer tissues was decreased. The present results suggested that a decrease in the expression level of ARID1A may promote the occurrence and development of gastric cancer. Furthermore, statistical analyses suggested a correlation between certain clinicopathological parameters and the protein expression level of ARID1A in patients with gastric cancer. In line with our previous study (14), it was identified that the protein expression level of ARID1A in gastric cancer tissues was associated with depth of tumor invasion, which is correlated with poor prognosis. The present results suggested that a decrease in the protein expression level of ARID1A indicated poor prognosis of gastric cancer.

Harada et al (22) observed that the protein expression level of PIK3CA was not associated with any clinicopathological features. However, the present study suggested that $60.7 \%(182 / 300)$ of gastric cancers exhibited an increased protein expression level of PIK3CA. The protein expression level increased in gastric cancer tissues compared with adjacent normal tissues. Furthermore, the protein expression level of PIK3CA was identified to be correlated with tumor differentiation, lymphatic metastasis and depth of invasion; however, our previous study suggested that the protein expression level of PIK3CA was associated only with the depth of invasion (14). The present study suggested that the protein expression level of PIK3CA may be associated with the development of gastric cancer, consistently with previous findings $(23,24)$.

Furthermore, previous genome-wide studies identified that the mutation rate of ARID1A was the highest in numerous types of cancer, including ovarian, endometrial, breast and colorectal cancer $(25,26)$. The frequency of ARID1A mutations in gastric cancer was identified to be $8-33 \%$ (27), and the most common types of mutations were frameshift and nonsense mutations (28). The present results suggested that $62.1 \%(36 / 58)$ of patients with gastric cancer exhibited mutations in ARID1A, including 44.4\% (16/36) nonsense mutations and $55.6 \%$ (20/36, including 2 cases of deletion mutations) missense mutations. The tissues exhibiting missense mutations presented abnormal protein expression levels of ARID1A, as determined by immunohistochemical examination, with altered protein expression level of ARID1A and increased protein expression level of PIK3CA. The sample selection method may have affected the SNP genotyping results, leading to an increased rate of ARID1A mutation in the gastric cancer group compared with previous studies. The mutation rate of ARID1A in EBVaGC (78.6\%) was increased compared with EBVnGC (46.7\%), although EBV-negative pediatric Burkitt lymphoma exhibited an increase in the mutation rate of ARID1A (29). In the present study, SNP genotyping assays suggested that $13.8 \%$ (8/58) of gastric cancer tissues exhibiting PIK3CA mutations were EBVaGC, whereas, no PIK3CA mutation was identified in adjacent normal tissues. Notably, tissues presenting PIK3CA mutations additionally exhibited ARID1A mutations. Although previous studies identified that ARID1A and PIK3CA may be simultaneously mutated in ovarian clear carcinomas (30), the concurrent mutations of ARID1A and PIK3CA in gastric cancer have not been previously observed, to the best of the authors' knowledge. The present results suggested that EBV, ARID1A and PIK3CA may be associated in the development and progression of gastric cancer via a specific mechanism. Fang et al (2) suggested that tumors located in the second third of the stomach exhibiting mutations in PIK3CA are susceptible to EBV infection. Additionally, it was demonstrated that EBV may cause gastric carcinoma by inducing abnormal DNA methylation (31). These previous studies suggested that EBV may promote abnormal DNA methylation in gastric cancer with mutations in PIK3CA. A previous study demonstrated that mutations in PIK3CA may promote the activation of the PI3K/AKT pathway (32) and that the PI3K/AKT signaling pathway may serve an important role in numerous processes, including proliferation, angiogenesis and apoptosis $(31,33)$. Furthermore, multiple studies have suggested that the phosphorylation levels of AKT and the activation of PI3K are negatively associated with the expression level of ARID1A $(34,35)$. These previous studies suggested that ARID1A may be involved in the regulation of the PI3K/AKT pathway in gastric cancer. Additionally, SWI/SNF complex, containing ARID1A, was identified to interact with the promoter sequence of PIK3CA, 1,127 to 946 nucleotides upstream of ARID1A transcription starting site (28). In the present study, it was hypothesized that PIK3CA may be a direct target gene of ARID1A in the PI3K/AKT pathway, and that the loss of ARID1A in gastric cancer may result in the loss of the inhibitory ability of PIK3CA, thus activating the PI3K/AKT pathway, promoting tumor progression. Collectively, the present results suggested that EBV, PIK3CA and ARID1 A may be associated in gastric cancer via the PI3K/AKT pathway. However, the specific mechanism underlying the regulation of these factors requires further investigation.

EBVaGC is a heterogeneous disease (19). The immunohistochemistry of EBNA1 and LMP1 suggested that the majority of EBVaGCs were latent-I type (36). To the best of the authors' knowledge, no previous study demonstrated the interaction among EBV, ARID1A and PIK3CA. In the present study, the protein expression levels of ARID1A and PIK3CA in EBVaGC were investigated, and ARID1A and PIK3CA were identified to be negatively correlated in EBVaGC. However, the protein expression levels of ARID1A and PIK3CA did not exhibit a significant difference between EBVaGC and EBVnGC. In a previous study, mutations in PIK3CA were identified to be able to increase EBV susceptibility (2), although, to the best of the authors' knowledge, the association between EBV and ARID1A in gastric cancer has not yet been investigated. Further experiments are required to examine the effect of ARID1A mutations on the susceptibility to EBV. Furthermore, to analyze an increased number of samples may contribute in identifying the association among these three factors.

Collectively, EBV, ARID1A and PIK3CA may interact with each other and serve important roles in the development and progression of gastric cancer. The association between the expression levels of ARID1A and PIK3CA between groups was investigated in the present study; however, due to the limited number of samples collected, only a few cases exhibited a significant association. Collectively, due to the restrictions in the present experimental conditions, the present study had certain limitations. Further studies are required to examine an increased number of EBVaGC samples, and it is necessary 
to analyze the follow up data of the status of the patients, in order to investigate the association among EBV, ARID1A and PIK3CA at the molecular level.

\section{Acknowledgements}

Not applicable.

\section{Funding}

The study was supported by grants from The Hainan Natural Science Foundation of China (no. 20168316).

\section{Availability of data and materials}

The datasets used and/or analyzed during the current study are available from the corresponding author on reasonable request.

\section{Authors' contributions}

HL designed the experiments. HZ performed the experiments and analyzed the data. ST and XL analyzed the data, performed the literature search and drafted the manuscript. All authors read and approved the final manuscript.

\section{Ethics approval and consent to participate}

Consent was obtained prior to sample collection. The Central South University Xiangya School of Medicine Affiliated Haikou Hospital Ethical Review Committee approved all the experiments performed on human tissues (permit no. 2015013).

\section{Patient consent for publication}

Not applicable.

\section{Competing interests}

The authors declare that they have no competing interests.

\section{References}

1. Wu H, Lin W and Tsai K: Advances in molecular biomarkers for gastric cancer: miRNAs as emerging novel cancer markers. Expert Rev Mol Med 16: e1, 2014.

2. Fang WL, Huang KH, Lan YT, Lin $\mathrm{CH}$, Chang SC, Chen MH, Chao Y, Lin WC, Lo SS, Li AF, et al: Mutations in PI3K/AKT pathway genes and amplifications of PIK3CA are associated with patterns of recurrence in gastric cancers. Oncotarget 7: 6201-6220, 2016.

3. Camargo MC, Kim K, Matsuo K, Torres J, Liao LM, Morgan DR, Michel A, Waterboer T, Zabaleta J, Dominguez RL, et al: Anti-Helicobacter pylori antibody profiles in epstein-Barr virus (EBV)-positive and EBV-negative gastric cancer. Helicobacter 21: 153-157, 2016.

4. Camargo MC, Bowlby R, Chu A, Pedamallu CS, Thorsson V, Elmore S, Mungall AJ, Bass AJ, Gulley ML and Rabkin CS: Validation and calibration of next-generation sequencing to identify Epstein-Barr virus-positive gastric cancer in The cancer genome atlas. Gastric Cancer 19: 676-681, 2016.

5. Liu Y, Yang W, Pan Y, Ji J, Lu Z and Ke Y: Genome-wide analysis of Epstein-Barr virus (EBV) isolated from EBV-associated gastric carcinoma (EBVaGC). Oncotarget 7: 4903-4914, 2016.
6. Zhang L, Wang C, Yu S, Jia C, Yan J, Lu Z and Chen J: Loss of ARID1A expression correlates with tumor differentiation and tumor progression stage in pancreatic ductal adenocarcinoma. Technol Cancer ResTreat 17: 1-10, 2018.

7. Wang S, Nassour I, Xiao S, Zhang S, Luo X, Lee J, Li L, Sun X, Nguyen LH, Chuang JC, et al: SWI/SNF component ARID1A restrains pancreatic neoplasia formation. Pancreas: doi:10.1136/ gutjnl-2017-315490.

8. Shen J, Peng Y, Wei L, Zhang W, Yang L, Lan L, Kapoor P, Ju Z, Mo Q, Shih IM, et al: ARID1A deficiency impairs the DNA damage checkpoint and sensitizes cells to PARP inhibitors. Cancer Discov 5: 752-767, 2015.

9. Mathur R, Alver BH, San Roman AK, Wilson BG, Wang X, Agoston AT, Park PJ, Shivdasani RA and Roberts CW: ARID1A loss impairs enhancer-mediated gene regulation and drives colon cancer in mice. Nat Genet 49: 296-302, 2016.

10. Abe H, Kaneda A and Fukayama M: Epstein-Barr virusassociated gastric carcinoma: Use of host cell machineries and somatic gene mutations. Pathobiology 82: 212-223, 2015.

11. Wang L, Shan L, Zhang S, Ying J, Xue L, Yuan Y, Xie Y and Lu N: PIK3CA gene mutations and overexpression: Implications for prognostic biomarker and therapeutic target in chinese esophageal squamous cell carcinoma. PLoS One 9: e103021, 2014.

12. Park JH, Ryu MH, Park YS, Park SR, Na YS, Rhoo BY and Kang YK: Successful control of heavily pretreated metastatic gastric cancer with the mTOR inhibitor everolimus (RAD001) in a patient with PIK3CA mutation and pS6 overexpression. BMC Cancer 15: 119, 2015.

13. Wang Q, Shi YL, Zhou K, Wang LL, Yan ZX, Liu YL, Xu LL, Zhao SW, Chu HL, Shi TT, et al: PIK3CA mutation confer resistance to first-line chemotherapy in colorectal cancer. Cell Death Dis 9: 739, 2018.

14. Li Hong, Tan Shun, Xing Lu, Li Qian and Zhou Huan: Expression and significance of EBV, ARID1A and PIK3CA gene in gastric cancer. Hainan Med J 29: 1344-1347, 2018.

15. Liu P, Qin K, Wu K, Shi H and Zhang L: The expression and significance of HAND2 in the myenteric plexus of the colon in patients with Hirschsprung's disease. Guangdong Med J 34: 1513-1515, 2013 (In Chinese).

16. Wang K, Kan J, Yuen ST, Shi ST, Chu KM, Law S, Chan TL, Kan Z, Chan ASY, Tsui WY, et al: Exome sequencing identifies frequent mutation of ARID1A in molecular subtypes of gastric cancer. Nature Genetics 43: 1219-1223, 2011.

17. Kanda T, Furuse Y, Oshitani H and Kiyono T: Highly efficient CRISPR/Cas9-mediated cloning and functional characterization of gastric cancer-Derived epstein-Barr virus strains. J Virol 90: 4383-4393, 2016

18. Zhang L, Wu H, Sun G, Xu X, Sun X and Cao L: Trichloromethane fraction of Incarvillea compacta induces lytic cytotoxicity and apoptosis in Epstein-Barr virus-positive gastric cancer AGS cells. BMC Complem Altern Med 16: 344-354, 2016.

19. Gulley ML: Genomic assays for Epstein-Barr virus-positive gastric adenocarcinoma. Exp Mol Med 47: e134, 2015.

20. Wang DD: Decreased expression of the ARID1A gene Is associated with poor prognosis in primary gastric Cancer. PLoS One 7: e40364, 2012.

21. Luchini C, Veronese N, Solmi M, Cho H, Kim J, Chou A, Gill AJ, Faraj SF, Chaux A, Netto GJ, et al: Prognostic role and implications of mutation status of tumor suppressor gene ARID1A in cancer: A systematic review and meta-analysis. Oncotarget 6: 39088-39097, 2015.

22. Harada K, Baba Y, Shigaki H, Ishimoto T, Miyake K, Kosumi K, Tokunaga R, Izumi D, Ohuchi M, Nakamura K, et al: Prognostic and clinical impact of PIK3CA mutation in gastric cancer: Pyrosequencing technology and literature review. BMC Cancer 16: 400-407, 2016.

23. Ibarrola-Villava M, Llorca-Cardenosa MJ, Tarazona N, Mongort C, Fleitas T, Perez-Fidalgo JA, Rosello S, Navarro S, Ribas G and Cervantes A: Deregulation of ARID1A, CDH1, cMET and PIK3CA and target-related microRNA expression in gastric cancer. Oncotarget 6: 26935-26945, 2015.

24. Jang SH, Kim KJ, Oh MH,Lee JH and Lee HJ: Clinicopathological significance of elevated PIK3CA expression in gastric cancer. J Gastric Cancer 16: 85-92, 2016.

25. Wu Y, Gu Y, Guo S, Dai Q and Zhang W: Expressing status and correlation of ARID1 A and histone H2B on breast cancer. Biomed Res Int 2016: 759387, 2016.

26. Wei X, Wang DS, Xi SY, Wu WJ, Chen DL, Zeng ZL, Wang RY, Huang YX, Jin Y, Wang F, et al: Clinicopathologic and prognostic relevance of ARID1A protein loss in colorectal cancer. World J Gastroenterol 20: 18404-18412, 2014. 
27. Lee D, Yu EJ, Ham IH, Hur H and Kim YS: AKT inhibition is an effective treatment strategy in ARID1A-deficient gastric cancer cells. Onco Targrts Ther 10: 4153-4159, 2017.

28. Zhang Q, Yan HB, Wang J, Cui SJ, Wang XQ, Jiang YH, Feng L, Yang PY and Liu F: Chromatin remodeling gene AT-rich interactive domaincontaining protein $1 \mathrm{~A}$ suppresses gastric cancer cell proliferation by targeting PIK3CA and PDK1. Oncotarget 7: 46127-46141, 2016.

29. Giulino-Roth L, Wang K, Macdonald TY, Mathew S, Tam Y, Cronin MT, Palmer G, Lucena-Silva N, Pedrosa F, Pedrosa M, et al: Targeted genomic sequencing of pediatric Burkitt lymphoma identifies recurrent alterations in antiapoptotic and chromatin-remodeling genes. Blood 120: 5181-5184, 2012.

30. Wu R, Wang T and Shih IeM: The emerging roles of ARID1A in tumor suppression. Cancer Biol Ther 15: 655-664, 2014.

31. Böger C, Krüger S, Behrens HM, Bock S, Haag J, Kalthoff H, and Röcken C: Epstein-Barr virus-associated gastric cancer reveals intratumoral heterogeneity of PIK3CA mutations. Ann Oncol 28: 1005-1014, 2017.

32. Kaneda A, Matsusaka K, Aburatani H and Fukayama M: Epstein-Barr virus infection as an epigenetic driver of tumorigenesis. Cancer Res 72: 3445-3450, 2012.

33. Suzuki Y, Enokido Y, Yamada K, Inaba M, Kuwata K, Hanada N, Morishita T, Mizuno S and Wakamatsu N: The effect of rapamycin, NVP-BEZ235, aspirin, and metforminon PI3K/AKT/mTOR signaling pathway of PIK3CA-related overgrowth spectrum (PROS). Oncotarget 8: 45470-45483, 2017.
34. Wang XY, Huang YX, Guo RH, Liu YB, Qian YY, Liu D, Dai XJ, Wei Z, Jin F and Liu YQ: Clinicopathological significance of ROCK1 and PIK3CA expression in nasopharyngeal carcinoma. Exp Ther Med 13: 1064-1068, 2017.

35. Zhang Q, Yan HB, Wang J, Cui SJ, Wang XQ, Jiang YH, Feng L, Yang P Y and Liu F: Chromatin remodeling gene AT-rich interactive domain-containing protein $1 \mathrm{~A}$ suppresses gastric cancer cell proliferation by targeting PIK3CA and PDK1. Oncotarget 7: 46127-46141, 2016.

36. Sivachandran N, Dawson CW, Young LS, Liu FF, Middeldorp J and Frappier L: Contributions of the epstein-Barr virus EBNA1 protein to gastric carcinoma. J Virol 86: 60-68, 2011.

(i) (5) This work is licensed under a Creative Commons Attribution-NonCommercial-NoDerivatives 4.0 International (CC BY-NC-ND 4.0) License. 